\title{
Combination of gemcitabine and erlotinib inhibits recurrent pancreatic cancer growth in mice via the JAK-STAT pathway
}

\author{
LIWEN CHEN $^{1 *}$, DING'AN ZHOU ${ }^{2 *}$, ZHEHAO LIU $^{1}$, XINHAO HUANG $^{1}$, QIANFAN LIU ${ }^{1}$, \\ YIPING $\mathrm{KANG}^{3}$, ZILI CHEN ${ }^{1}$, YUNTAO GUO ${ }^{1}$, HAITAO ZHU ${ }^{1,2}$ and CHENGYI SUN ${ }^{1}$ \\ ${ }^{1}$ Department of Hepatobiliary Surgery and ${ }^{2}$ Clinical Research Center, The Affiliated Hospital of Guizhou Medical University; \\ ${ }^{3}$ Clinical Medical College, Guizhou Medical University, Guiyang, Guizhou 550001, P.R. China
}

Received May 27, 2017; Accepted December 18, 2017

DOI: $10.3892 /$ or.2018.6198

\begin{abstract}
Compared to single gemcitabine treatment, the combination of gemcitabine and erlotinib has shown effective response in patients with locally advanced or metastatic pancreatic cancer. However, the combination therapy has not proven effective in patients with pancreatic cancer after R0 or $\mathrm{R} 1$ resection. In the present study, a nude mice model of orthotopic xenotransplantation after tumor resection was established using pancreatic cancer cell lines, BxPC-3 and PANC-1. Mice were divided in four groups (each with $n=12$ ) and were treated as follows: the control group received a placebo via intraperitoneal injection (i.p.), while the other three groups were treated with gemcitabine $(50 \mathrm{mg} / \mathrm{kg}$ i.p., twice a week), erlotinib (50 mg/kg oral gavage, once every three days), and combined treatment of gemcitabine and erlotinib, respectively. The treatment lasted for 21 days, after which all mice were sacrificed and tumors were examined ex vivo. We determined that the combination of gemcitabine and erlotinib inhibited recurrent tumor growth and induced apoptosis in vivo by downregulating phosphorylation levels of JAKs and STATs, which in turn downregulated the downstream proteins HIF-1 $\alpha$ and cyclin D1, and upregulated caspase-9 and caspase-3 expression. To sum up, the combination of gemcitabine with
\end{abstract}

Correspondence to: Dr Haitao Zhu or Dr Chengyi Sun, Department of Hepatobiliary Surgery, The Affiliated Hospital of Guizhou Medical University, 28 Gui yi Road, Guiyang, Guizhou 550001, P.R. China

E-mail: 754667317@qq.com

E-mail: chengyisun@medmail.com.cn

*Contributed equally

Abbreviations: i.p., intraperitoneal injection; PC, pancreatic cancer; EGFR, epidermal growth factor receptor; TKI, tyrosine kinase inhibitor; JAK, Janus kinase; STAT, signal transducers and activators of transcription; TUNEL, terminal deoxynucleotidyl transferase-mediated dUTP nick end labeling; TBST, TBS containing $0.1 \%$ Tween-20; CDK4, cyclin-dependent kinase 4; HIF-1, hypoxia-inducible factor-1

Key words: pancreatic cancer, gemcitabine, erlotinib, JAK-STAT pathway erlotinib was effective in treating patients with pancreatic cancer after $\mathrm{R} 0$ or $\mathrm{R} 1$ resection.

\section{Introduction}

Pancreatic cancer (PC) is the 10th most commonly diagnosed cancer in the world. In 2013, it was the fourth leading cause of deaths in the United States (1). The 5-year overall survival rate in patients with $\mathrm{PC}$ is roughly 5\%. Currently, surgery is considered to be the most effective treatment for these patients (2). However, only $20 \%$ of patients diagnosed with pancreatic cancer are eligible to undergo the surgery (3). For most patients with PC, long-term survival rates cannot be improved even after undergoing radical resection. Moreover, chemotherapeutic drugs, such as gemcitabine or fluorouracil/ folinic acid have been demonstrated to be effective against PC. Single-agent gemcitabine is a first-line drug for treating patients with advanced pancreatic cancer or patients after $\mathrm{R} 0$ or R1 resection $(4,5)$. However, this treatment approach produces only modest improvements (6).

Gemcitabine is a deoxycytidine analog and its cytotoxic activity is based on several activities of DNA synthesis $(4,7)$. To date, gemcitabine remains the cornerstone of neo-adjuvant, adjuvant and palliative therapy for PC (7). Along with gemcitabine, erlotinib is the first additional drug used to improve the overall survival of patients with advanced pancreatic cancer (4). Erlotinib is an oral small-molecule epidermal growth factor receptor (EGFR) tyrosine kinase inhibitor (TKI), which can block the signal transduction pathway essential for cancer differentiation, proliferation, apoptosis, angiogenesis, invasion, and metastasis (8). The combination of gemcitabine and erlotinib has been reasonably effective in treating patients with locally advanced or metastatic pancreatic cancer $(4,9)$. However, the mechanism of action of gemcitabine in combination with erlotinib in pancreatic cancer remains unknown and requires further investigation.

The Janus kinase/signal transducers and activators of transcription (JAK-STAT) pathway is the principal signaling mechanism for a wide array of cytokines and growth factors. JAK activation stimulates cell proliferation, differentiation, migration and apoptosis in mammals (10). The activated JAKs subsequently phosphorylate additional targets, including both receptors and the major substrates, STATs. STATs are latent 
transcription factors that reside in the cytoplasm until activated. The seven mammalian STATs bear a conserved tyrosine residue near the C-terminus that is phosphorylated by JAKs. This phosphotyrosine permits the dimerization of STATs through interaction with a conserved SH2 domain (10), which then enter the nucleus. Once in the nucleus, dimerized STATs bind specific regulatory sequences to activate or suppress transcription of target genes. Furthermore, the activation of the JAK-STAT pathway was determined to be associated with human pancreatic cancer (11).

The present study investigated the effectiveness of gemcitabine in combination with erlotinib on recurrent pancreatic cancer growth in vivo. In this study, a nude mice model of orthotopic xenotransplantation after tumor resection was established using pancreatic cancer cell lines, BxPC-3 and PANC-1. Consequently, mice were divided in different experimental groups and treated for 3 weeks, after which the recurrent tumor tissues were dissected and analyzed ex vivo.

\section{Materials and methods}

Cell lines. The human pancreatic cancer cell lines BxPC-3 and PANC-1 were purchased from the Chinese Academy of Sciences (Shanghai, China) and the American Type Culture Collection (ATCC, Manassas, VA, USA), respectively. Human pancreatic adenocarcinoma cell lines BxPC-3, and PANC-1 were both cultured in DMEM (Gibco, Thermo Fisher Scientific, Inc., Waltham, MA, USA), supplemented with $10 \%$ fetal bovine serum (FBS; Gibco, Thermo Fisher Scientific, Inc.) in a humidified atmosphere containing $5 \% \mathrm{CO}_{2} / 95 \%$ air at $37^{\circ} \mathrm{C}$. For the animal experiments, the cells were trypsinized, resuspended in Matrigel (BD Biosciences, Franklin Lakes, NJ, USA) at a concentration of $1 \times 10^{7}$ cells $/ 30 \mu 1$, and stored on ice until injection.

Drugs. Erlotinib (Tarceva) was purchased from Roche Pharmaceutical Ltd. (Basel, Switzerland) as a fine powder, and was consequently dissolved in distilled water containing 6\% (w/v) Captisol (Aoke Biotechnology, Ltd., Qingdao, China). Gemcitabine (Gemzar) was purchased from Eli Lilly and Company (Neuilly sur Seine, France) and dissolved in $0.9 \%$ saline solution.

Orthotopic xenotransplantation of human pancreatic cancer cells and tumor resection. Four-week-old female nude mice weighing 18-20 g were obtained from Beijing HuaFuKang Bioscience Co., Ltd. (Beijing, China). All the animals were housed (acclimatized for 10 days in a sterile environment, in which bedding, food, and water were autoclaved) in an environment with a temperature of $22 \pm 1^{\circ} \mathrm{C}$, relative humidity of $50 \pm 1 \%$ and a light/dark cycle of $12 / 12 \mathrm{~h}$. All animal studies (including the mice euthanasia procedure) were performed in compliance with the regulations and guidelines of Guizhou Medical University Institutional Animal Care and conducted according to the AAALAC and the IACUC guidelines.

Mice were divided in four groups (each with $n=12$ ). After establishment of orthotopic xenotransplantation of human pancreatic cancer cells after tumor resection (12) they were treated as follows: the control group received a placebo ( $0.9 \%$ saline, every three days) via intraperitoneal injec- tion (i.p.); the administration of gemcitabine was performed via i.p. in the gemcitabine group (group G) $(50 \mathrm{mg} / \mathrm{kg}$ i.p., twice a week) and erlotinib was administered by oral gavage in the erlotinib group (group E) $(50 \mathrm{mg} / \mathrm{kg}$ oral gavage, once every three days). The mode of drug administration in the gemcitabine-erlotinib combination group ( $\mathrm{E}+\mathrm{G}$ group) was performed by oral gavage of erlotinib $(50 \mathrm{mg} / \mathrm{kg})$ on the first day and i.p. of gemcitabine $(50 \mathrm{mg} / \mathrm{kg}$ ) was administered the next day. On the third day, the mice were left to rest and then administration of the drugs continued in the next three days. The treatments lasted for 21 days, after which all mice were sacrificed and the tumors were examined ex vivo according to a previously described method (24). A recurrent tumor was defined as a new tumor mass found during relaparotomy which was performed four weeks after the first surgery, removing all the tumor mass that was in the area where we inoculated.

Physical measurements of tumor volume. At necropsy, the tumors were dissected away from the normal pancreas and their measured tumor volume was calculated as: volume $\left(\mathrm{mm}^{3}\right)$ $=\left(\right.$ length $\mathrm{x}$ width $\left.{ }^{2}\right) / 2(13)$.

Terminal deoxynucleotidyl transferase-mediated dUTP nick end labeling (TUNEL) assay. Paraffin blocks were cut into slices with $4-\mu \mathrm{m}$ thickness. A TUNEL assay was conducted using a TUNEL apoptosis detection kit (Nanjing, KeyGen Biotech, Co., Ltd., Jiangsu, China) following the manufacturer's protocol. Paraffin sections were incubated with proteinase $\mathrm{K}$ at $37^{\circ} \mathrm{C}$ for $30 \mathrm{~min}$. Endogenous peroxidase activity was blocked by incubation with $3 \%$ hydrogen peroxide in methanol. Positive control sections were treated with DNase I $50 \mathrm{U} / \mu \mathrm{l}$, while the negative control sections were incubated with label solution (without the terminal deoxynucleotidyl transferase enzyme). All the other sections were incubated with TUNEL reaction mixture at $37^{\circ} \mathrm{C}$ for $1 \mathrm{~h}$ in a humidity chamber. The conjugated horseradish peroxidase was visualized with diaminobenzidine. Finally, the sections were counterstained with hematoxylin and the images were captured in a positive position under the microscope BX51 (Olympus Corp., Tokyo, Japan). The results were expressed as the percentage of TUNEL-positive cells per x40 magnification. A total of ten x40 fields were examined from three tumors in each of the treatment groups.

Western blot analysis. The tumor tissue was first laced in a homogenizer, and then on ice for homogenization with RIPA buffer containing a protease inhibitor cocktail and a phosphatase inhibitor cocktail (Beijing Solarbio Science \& Technology, Co., Ltd., Beijing, China). The cell lysates were centrifuged and boiled with SDS loading buffer. Then the protein samples were subjected to SDS-polyacrylamide gel electrophoresis (Bio-Rad Laboratories, Inc., Hercules, CA, USA) and electrophoretically transferred to $0.45-\mu \mathrm{m}$ polyvinylidene difluoride membranes (Millipore, Billerica, MA, USA). After incubation with $5 \%$ non-fat dry milk in TBS containing $0.1 \%$ Tween-20 (TBST) for $1 \mathrm{~h}$, the membranes were washed once with TBST and probed with the indicated primary antibodies overnight at $4^{\circ} \mathrm{C}$. The following primary antibodies were used: antiJAK1 (rabbit polyclonal; cat. no. AF5012), phospho-JAK1 $\left(\mathrm{Tyr}^{1022}\right)$ (rabbit polyclonal; cat. no. AF2012), anti-JAK2 (rabbit polyclonal; cat. no. AF6022), phospho-JAK2 (Tyr ${ }^{221}$ ) 
Table I.Apoptotic index in recurrent tumors of both BxPC-3 and PANC-1 cells.

\begin{tabular}{lcccrr}
\hline Cell line & N group $($ mean $\pm \mathrm{SD})$ & G group $($ mean $\pm \mathrm{SD})$ & E group $($ mean $\pm \mathrm{SD})$ & $\mathrm{E}+\mathrm{G}$ group $($ mean $\pm \mathrm{SD})$ & $\mathrm{P}$-value \\
\hline BxPC-3 & $9.5 \pm 3.63$ & $47.3 \pm 6.11$ & $29.1 \pm 4.01$ & $71.34 \pm 4.55$ & $\mathrm{P}<0.05^{\mathrm{a}}$ \\
PANC-1 & $11.7 \pm 1.77$ & $45.3 \pm 6.15$ & $41.4 \pm 5.50$ & $77.6 \pm 4.65$ & $\mathrm{P}<0.05^{\mathrm{a}}$ \\
\hline
\end{tabular}

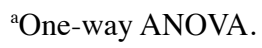

(rabbit polyclonal; cat. no. AF3023), anti-JAK3 (mouse monoclonal; cat. no. BF0256), phospho-JAK3 (Tyr ${ }^{981}$ ) (rabbit polyclonal; cat. no. AF8160), phospho-STAT1 (Tyr ${ }^{701}$ ) (rabbit polyclonal; cat. no. AF3300) and anti-STAT1 (rabbit polyclonal; cat. no. AF6300) were all purchased from Affinity Biosciences (Cambridge, UK); anti-STAT3 (rabbit monoclonal; cat. no. ab76315), and anti-pSTAT3 Try ${ }^{705}$ (rabbit monoclonal; cat. no. ab68153) were obtained from Abcam (Cambridge, MA, USA); anti-HIF-1 $\alpha$ (rabbit polyclonal; cat. no. BS3514) and anticyclin D1 (rabbit polyclonal; cat. no. BS6532) were purchased from Bioworld Technology Co., Ltd. (Nanjing, China); anti-p53 (rabbit, monoclonal; cat. no. 2527) was acquired from Cell Signaling Technology, Inc.(Danvers, MA, USA); while GAPDH was purchased from EarthOx Life Sciences (Millbrae, CA, USA). All the primary antibodies were diluted 1:500-1:1,000 in primary antibody dilution buffer (Beyotime Institute of Biotechnology, Haimen, China). The membranes were washed three times with TBST and incubated with a 1:1,000 dilution of horseradish peroxidase-conjugated anti-rabbit antibodies polyclonal antibodies (cat. no. SP-9001; ZSGB-BIO, Beijing, China) for $1 \mathrm{~h}$. After being washed three times with TBST, the membranes were incubated with enhanced chemiluminescence substrate (ECL; Millipore) for $5 \mathrm{~min}$. Chemiluminescent signals were visualized by exposing the membrane to ChemiDoc ${ }^{\text {тм тм }}$ XRS+ (Bio-Rad Laboratories, Inc.). Immunoblot analyses were performed at least three times for all the antibodies. The immune complexes were analyzed with ImageJ software $(\mathrm{NIH}$, Bethesda, MD, USA) which assessed the band intensity and relative protein abundance.

Immunohistochemical staining. The recurrent tumor tissues were fixed in $10 \%$ formalin at $4^{\circ} \mathrm{C}$ for $24 \mathrm{~h}$ and embedded in paraffin for immunohistochemistry analyses. The sections covered with tumor tissues were deparaffinized and rehydrated using xylene and an ethanol gradient. The following primary antibodies were used: anti-caspase-9 (rabbit polyclonal; bs-0049R) and anti-caspase-3 (rabbit polyclonal; bs-0081R), both purchased from BIOSS (Beijing, China). The primary antibodies were diluted 1:200 in PBS. Sections were incubated with active-caspase-9- and active-caspase-3 polyclonal antibodies (pAb), as well as a 1:3,000 dilution of horseradish peroxidase-conjugated anti-rabbit polyclonal antibodies (cat. no. 111-035-003; Jackson ImmunoResearch Laboratories, Inc. (West Grove, PA, USA). Finally, the sections were counterstained with hematoxylin and images were captured in a positive position under the microscope BX51 (Olympus Corp.). The staining intensity and percentage of positive cells were calculated and scored in sections. The staining intensity of the active-caspase-9 and active-caspase-3-positive cells was divided into four grades: 'negative (-)'-, '+', '++' and '+++' and scored as $0,1,2$ and 3, respectively. The positive cell percentages were divided into six categories: 'negative (-)', '1-20\%', '21-40\%', '41-60\%', '61-80\%' and '81-100\%' and scored as 0, 1, $2,3,4,5$ and 6 , respectively. Total scores of every visual field were determined using the formula: staining intensity scores of positive cells $\mathrm{x}$ percentage scores of positive cells $=$ total scores of each visual field.

Statistical analysis. Data were presented as the mean \pm SD. Comparisons among groups were performed by one-way ANOVA followed by the least significant difference (LSD) test. Comparisons between two groups were analyzed using Student's t-tests. $\mathrm{P}<0.05$ was considered to indicate a statistically significant result. All statistical analyses were performed using SPSS statistical software package, version 17.0 (SPSS, Inc., Chicago, IL, USA).

\section{Results}

The combination of gemcitabine and erlotinib inhibit recurrent tumor growth in mice. To investigate the effect of the combination of gemcitabine and erlotinib on recurrent tumor in vivo, an orthotopic tumor model was established and validated using BxPC-3 and PANC-1 cell lines (12). Briefly, recurrent and metastatic tumors were observed three weeks after tumor resection (Fig. 1). A significantly smaller tumor volume was observed in the group of mice treated with the combination of gemcitabine and erlotinib compared to the other groups $(\mathrm{P}<0.05$, for both cell lines) (Fig. 1). Hence, our data indicated that the combination of gemcitabine with erlotinib inhibited recurrent tumor growth more effectively than all the three other groups.

The combination of gemcitabine and erlotinib induces tumor apoptosis in mice. To assess the apoptotic feature in recurrent tumors among groups, apoptotic cells were counted after TUNEL staining. Positive apoptotic cells were significantly increased in the mice treated with gemcitabine (group G) and erlotinib (group E) compared with the control group (group N) $(\mathrm{P}<0.05$, for both cell lines) (Fig. 2). In addition, positive apoptotic cells were significantly increased in the group of mice treated with a combination of gemcitabine-erlotinib (group $\mathrm{E}+\mathrm{G}$ ) compared with the monotherapy groups (group $\mathrm{G}$ and group $\mathrm{E})(\mathrm{P}<0.05$, for both cell lines). The apoptotic index in the BxPC-3 and PANC-1 cells in group $\mathrm{N}$ was 9.5 \pm 3.63 and $11.7 \pm 1.77$, respectively. The ones in group $E$ were $29.1 \pm 4.01$ and $41.4 \pm 5.50$, respectively. The ones in group $\mathrm{G}$ were $47.3 \pm 6.11$ and $45.3 \pm 6.15$ ), respectively. The ones in group $E+G$ were $71.34 \pm 4.55$ and $77.6 \pm 4.65$ ), respectively (Table I). 

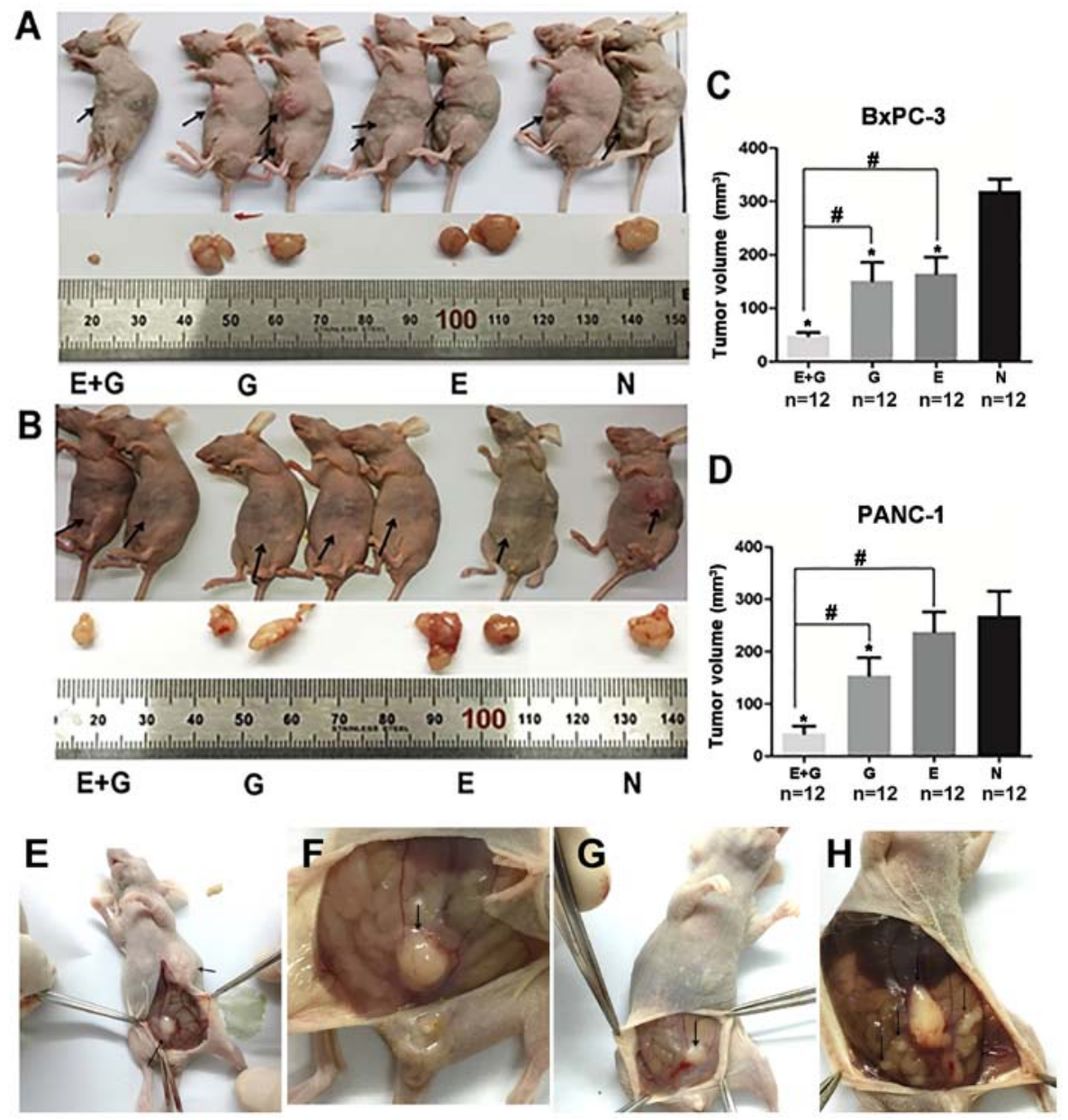

Figure 1. The combination of gemcitabine and erlotinib inhibits tumor growth in vivo. (A and B) The tumor volume data calculated by the standard tumor volume formula $\left[\left(\mathrm{x} \mathrm{w}^{2}\right) / 2\right]$. (C and $\left.\mathrm{D}\right)$ The tumor volume data calculated during the last day of treatment. ${ }^{*} \mathrm{P}<0.05$ indicates the negative controls vs. the treatment groups. "P $<0.05$ indicates the monotherapy $(\mathrm{E}$ or $\mathrm{G})$ vs. the combination $(\mathrm{E}+\mathrm{G})$ groups. $(\mathrm{E}-\mathrm{H})$ The metastatic tumors in the abdominal cavity were observed three weeks after tumor resection in mice bearing BxPC-3 and PANC-1 xenografts. E, erlotinib; G, gemcitabine; N, negative control.

A

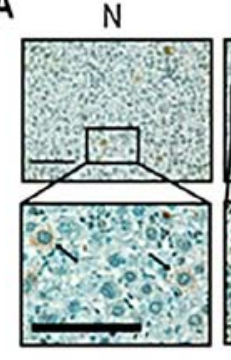

B

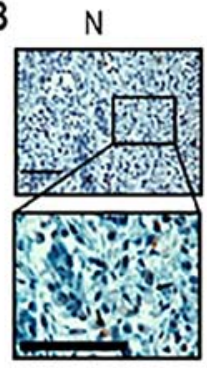

E

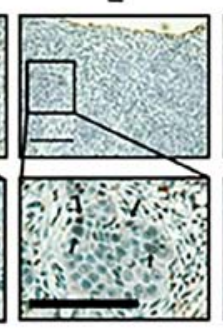

E

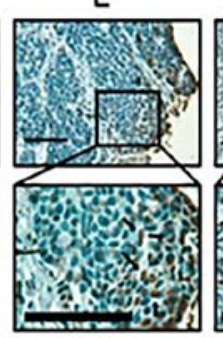

G

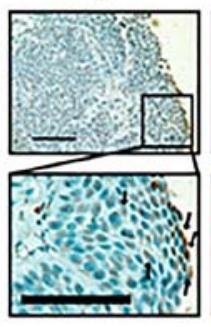

G

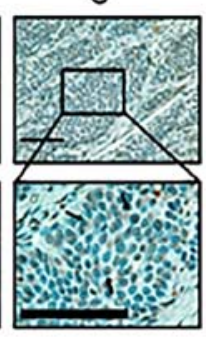

$E+G$

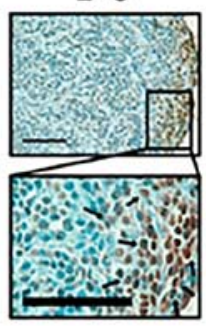

$E+G$

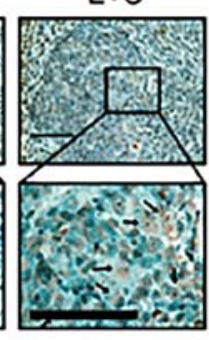

C

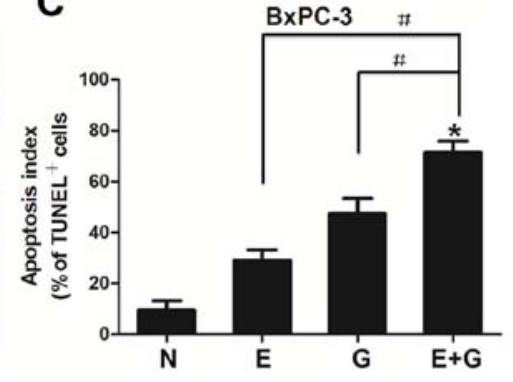

D

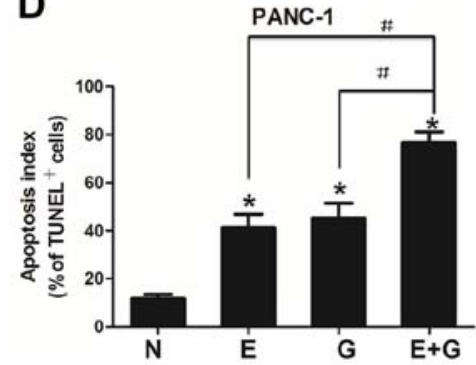

Figure 2. Tumor apoptosis detection in four experimental groups. (A and B) TUNEL in tumor tissue sections from different treatment groups revealed increased apoptosis in the monotherapy and in the combination treatment groups. Lower panels displayed higher magnification (original magnification, $\mathrm{x} 100$ ) of the boxed areas in the upper panels. Scale bars, $10 \mu \mathrm{m}$. (C and D) The apoptotic index was higher in the E+G group compared with the E or G group, in both BxPC-3 and PANC-1 cells. Quantification of TUNEL-positive cells in pancreatic cancer tumors. A total of ten $\mathrm{x} 40$ fields were examined from three tumors in each of the treatment groups. ${ }^{*} \mathrm{P}<0.05$ denotes the controls vs. the treatment groups. ${ }^{\prime} \mathrm{P}<0.05$ denotes the monotherapy $(\mathrm{E}$ or $\mathrm{G})$ vs. the combination $(\mathrm{E}+\mathrm{G})$ treatment groups. 

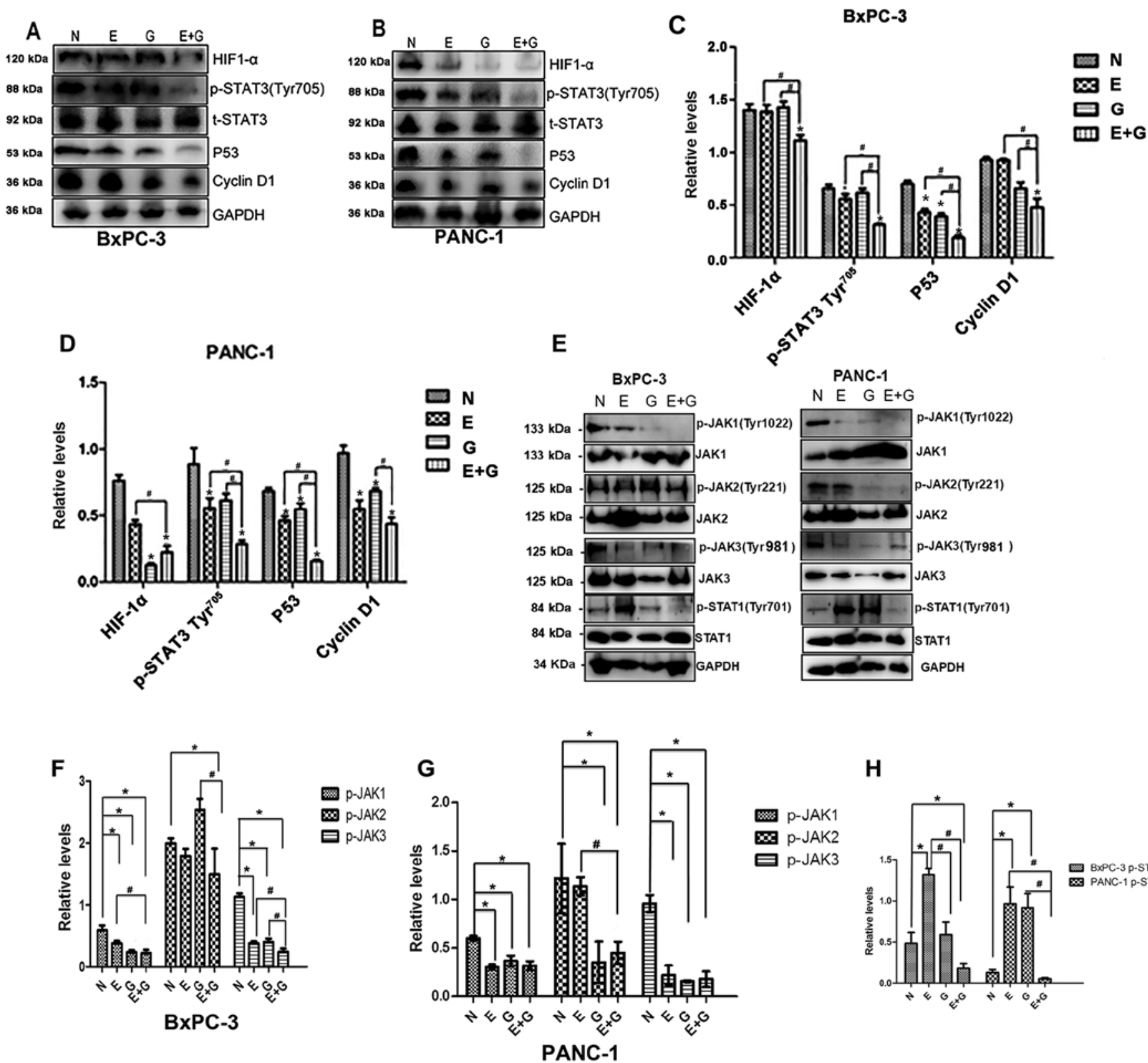

H

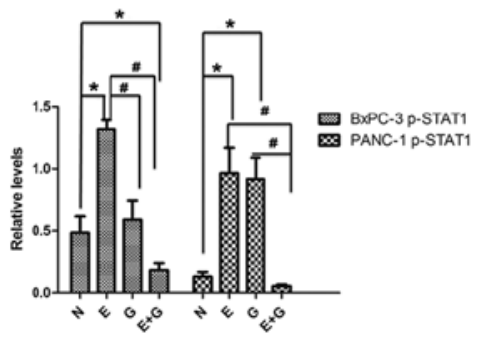

Figure 3. The gemcitabine-erlotinib (E+G) combination group inhibits the activity of the JAK-STAT pathway, as well as the expression of downstream HIF-1 $\alpha$, cyclin D1 and p53. (A and B) The protein levels of phosphorylated STAT3 Tyr $^{705}\left(\mathrm{p}-\mathrm{STAT} 3 \mathrm{Tyr}^{705}\right)$, HIF-1 $\alpha$, p53, cyclin D1 in BxPC-3 and PANC-1 cells were analyzed by western blotting, respectively. (C and D) The protein expression of p-STAT3 Tyr ${ }^{705}$, HIF-1 $\alpha$, p53, cyclin D1 in BxPC-3 and PANC-1 cells was calculated using one-way ANOVA. " $\mathrm{P}<0.05$ denotes the controls vs. the treatment groups. ${ }^{*} \mathrm{P}<0.05$ denotes the monotherapy (E or G) vs. the combination $(\mathrm{E}+\mathrm{G})$ groups. (E) Phosphorylation levels of JAK1 $\left(\mathrm{Tyr}^{1022}\right)$, JAK2 $\left(\mathrm{Tyr}^{221}\right)$, JAK3 $\left(\mathrm{Tyr}^{981}\right)$ and STAT3 $\left(\mathrm{Tyr}^{701}\right)$ in BxPC-3 and PANC-1 cells were analyzed by western blotting, respectively. ( $F$ and $\mathrm{G}$ ) The protein intensity of phospho-JAK1 (p-JAK1), p-JAK2 and p-JAK3 in BxPC-3 and PANC-1 cells was calculated using one-way ANOVA. ${ }^{*} \mathrm{P}<0.01 ;{ }^{*} \mathrm{P}<0.05$. $(\mathrm{H})$ The protein intensity of $\mathrm{p}-\mathrm{STAT} 1$ in $\mathrm{BxPC}-3$ and PANC-1 cells was assessed using one-way ANOVA. ${ }^{\text {"P }}<0.01$, ${ }^{*} \mathrm{P}<0.05$. E, erlotinib; G, gemcitabine.

The combination of gemcitabine and erlotinib suppresses the JAK-STAT signaling cascade. Since STAT3 activation is quite common in pancreatic cancer, and the phosphorylation of tyrosine residue 705 in the STAT3 protein is a crucial event for its activation (14), we examined the effects of gemcitabine combination with erlotinib on the phosphorylation of STAT3. As shown in Fig. 3A-D the phosphorylation of the tyrosine 705 residue in the STAT3 protein was significantly lower in group $\mathrm{E}$ and group $\mathrm{E}+\mathrm{G}$ compared to group $\mathrm{N}(\mathrm{P}<0.05)$. Phosphorylation levels of the STAT3 protein were decreased in group $\mathrm{E}+\mathrm{G}$ compared with group $\mathrm{E}$ and group $\mathrm{G}$, respectively
(Fig. 3A-D). In addition, the difference in the phosphorylation of STAT3 between groups N and group $\mathrm{G}$ was not significant in both cell lines (Fig. 3A-D).

Next, we evaluated the expression levels of p53, a molecule strongly associated with tumor growth and the phosphorylation of STAT3 and STAT1. Upstream of STAT3, the expression levels of JAK1, JAK2, JAK3 were also evaluated (Fig. 3). The expression level of p53 was significantly lower in group $\mathrm{E}+\mathrm{G}$ compared with group $\mathrm{G}$ or group $\mathrm{E}$, respectively, and it was significantly lower in group $\mathrm{G}$, group $\mathrm{E}$ and group $\mathrm{E}+\mathrm{G}$ when they were compared with group $\mathrm{N}$ in both cell lines. The 
A

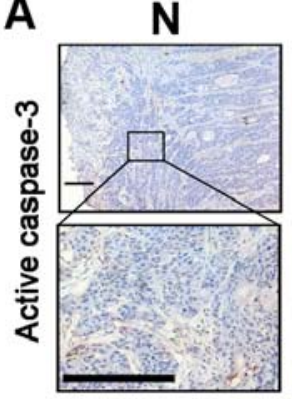

B

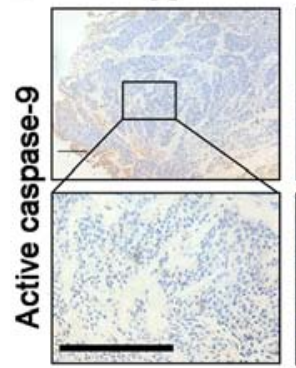

E

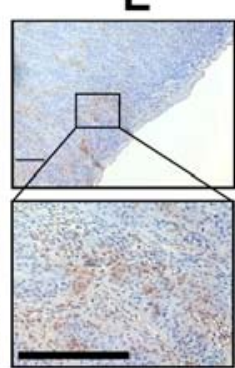

$\mathbf{E}$

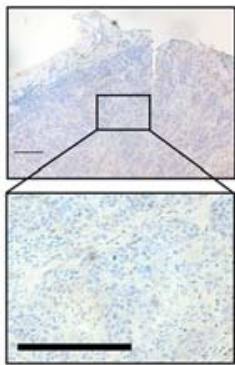

G

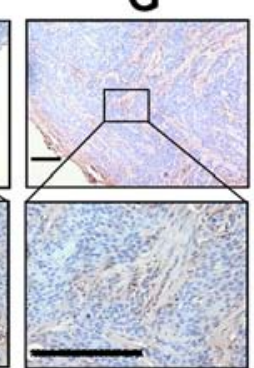

G

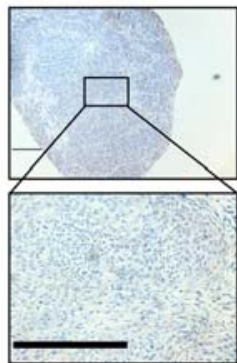

$E+G$

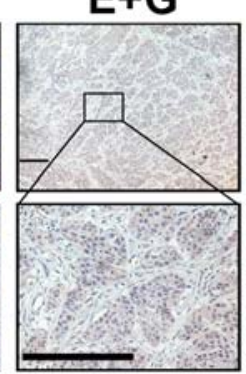

$E+G$

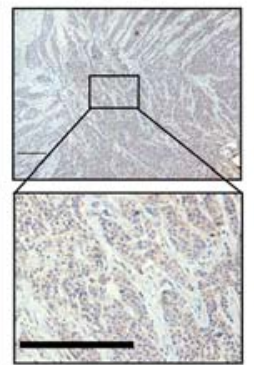

C

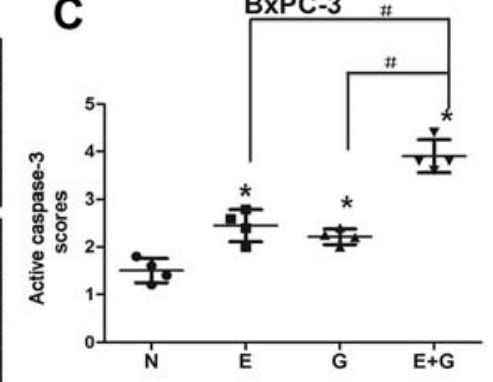

D

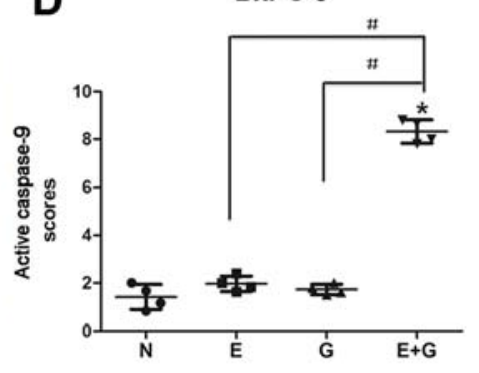

Figure 4. Gemcitabine in combination with erlotinib in recurrent tumors of BxPC-3 cells induces increased activity of caspase-3 and caspase-9. (A and B) Immunohistochemical staining of active caspase- 9 and active caspase- 3 in tumor tissue sections revealed active caspase- 9 activation by combination treatment and active-caspase-3 activation by either monotherapy and by combination treatment in the BxPC-3 cells. Lower panels displayed higher magnification (original magnification, $\mathrm{x} 400$ ) of the boxed areas in the upper panels. Scale bars, $10 \mu \mathrm{m}$. (C and D) Visual fields (4-6) in each section of irradiation were photographed. The staining intensity and the percentage of positive cells were calculated and scored in each section. The total scores of each visual field were first analyzed using the homogeneity of variance test, followed by the change of variable test. The scores were presented as the mean \pm SD. GraphPad Prism 5 was used to plot cartograms. ${ }^{*} \mathrm{P}<0.05$ denotes the controls vs. the treatment groups. ${ }^{\#} \mathrm{P}<0.05$ denotes the monotherapy $(\mathrm{E}$ or $\mathrm{G})$ vs. the combination $(\mathrm{E}+\mathrm{G})$ groups.

phosphorylation level of JAK1 was lower in group G, group E and group $\mathrm{E}+\mathrm{G}$ when they were compared with group $\mathrm{N}$ in both cell lines, and it was also lower when group E+G was compared with group E. The phosphorylation level of JAK2 was significantly lower in group $\mathrm{E}+\mathrm{G}$ when it was compared with group $\mathrm{N}$ or group $\mathrm{G}$ in recurrent tumors of the BxPC-3 cell line. While in recurrent tumors of the PANC-1 cell line, the phosphorylation level of JAK2 was significantly lower in group $\mathrm{E}+\mathrm{G}$ when it was compared with group $\mathrm{N}$ or group $\mathrm{E}$, and it was also lower in group $\mathrm{G}$ when it was compared with group N. As for the phosphorylation level of JAK3, it was lower in group $\mathrm{E}$, group $\mathrm{G}$ or group $\mathrm{E}+\mathrm{G}$ when they were compared with group $\mathrm{N}$ in both cell lines, respectively. In recurrent tumors of the BxPC-3 cell line, the phosphorylation level of JAK3 was lower in group E+G when compared with groups E, $\mathrm{G}$ and $\mathrm{N}$ respectively. The phosphorylation level of JAK3 was decreased in group $\mathrm{E}+\mathrm{G}$ when compared with groups $\mathrm{E}$ and $\mathrm{G}$, respectively. In recurrent tumors of PANC-1 cell line, the phosphorylation level of JAK3 in group $\mathrm{N}$ was higher than that of the other groups. However, no significant difference in the phosphorylation level of JAK3 was found between group E+G and group E or group $\mathrm{G}$, respectively. The phosphorylation level of STAT1 was significantly lower in group E+G when it was compared with group $\mathrm{N}$, group $\mathrm{E}$ or group $\mathrm{G}$, respectively. In addition, it was higher in group $\mathrm{E}$ when it was compared with group $\mathrm{N}$, group $\mathrm{G}$ and group $\mathrm{E}+\mathrm{G}$ in recurrent tumors of the BxPC-3 cell line, respectively. In the PANC-1 cell line recurrent tumors, the phosphorylation level of STAT1 was significantly lower in group $\mathrm{N}$ when it was compared with group $\mathrm{E}$ and group $\mathrm{G}$, respectively. Furthermore, it was also significantly lower in group $\mathrm{E}+\mathrm{G}$ when it was compared with group $\mathrm{E}$ and group $\mathrm{G}$ in recurrent tumors of the PANC-1 cell line, respectively.

Furthermore, we evaluated the expression level of cyclin D1 and HIF-1 $\alpha$, downstream proteins of STAT3. We found that the expression level of cyclin D1 was significantly lower in group $\mathrm{E}+\mathrm{G}$ than that in the monotherapy groups in recurrent tumors of the BxPC-3 cell line. The expression level of cyclin D1 was higher in group $\mathrm{N}$ when it was compared with the monotherapy groups. Cyclin D1 was lower in group $\mathrm{E}+\mathrm{G}$ when it was compared with group $\mathrm{G}$ in recurrent tumors of the PANC-1 cell line. The expression level of HIF-1 $\alpha$ was found to be significantly lower in group $\mathrm{E}+\mathrm{G}$ than in any of the other three groups and the expression level of HIF-1 $\alpha$ was found to be in recurrent tumors of the BxPC-3 cell line, significantly lower in group $\mathrm{G}$ than in group $\mathrm{N}(\mathrm{P}<0.05)$ in recurrent tumors of the PANC-1 cell line (Fig. 3A-D).

The combination of gemcitabine with erlotinib induces apoptosis of pancreatic cancer cells via caspase-dependent pathway. It was reported that reduction in STAT3 phosphorylation at tyrosine 705 residue could cause activation of caspase- 9 and caspase-3 (15). In the present study, we investigated the state of caspase- 9 and caspase- 3 by immunohistochemistry in all groups. Our data indicated that caspase- 9 and caspase- 3 were activated in mice bearing BxPC-3 and PANC-1 xenografts and treated with gemcitabine and erlotinib compared to the other groups (Figs. 4 and 5). These results indicated that the combination of gemcitabine and erlotinib induced apoptosis of pancreatic cancer cells via caspase-dependent pathway. 


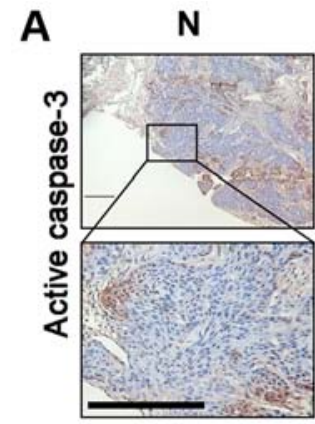

B

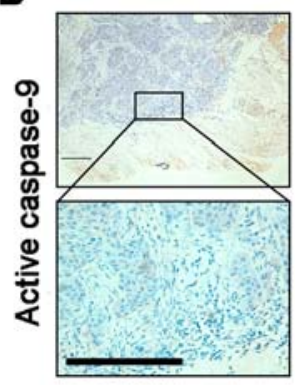

E

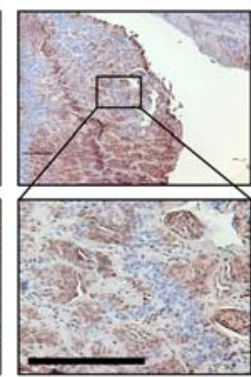

E

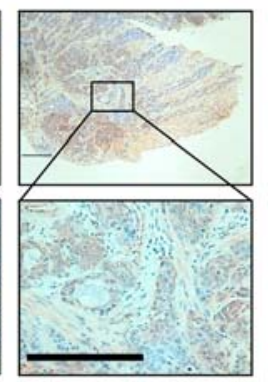

G

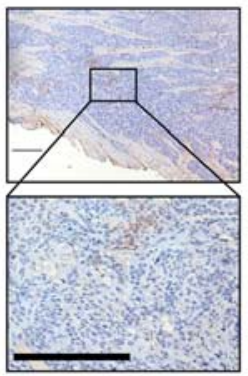

G

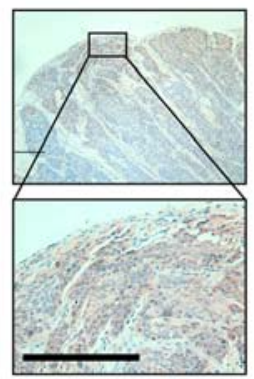

$E+G$

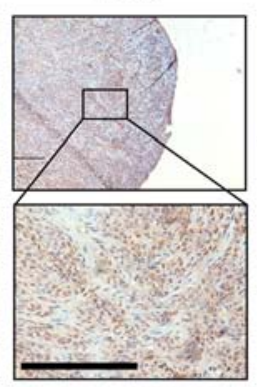

$E+G$

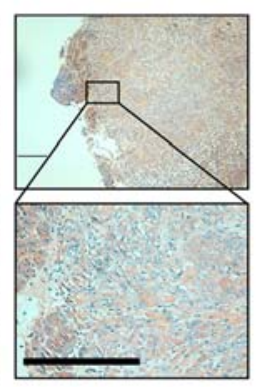

C

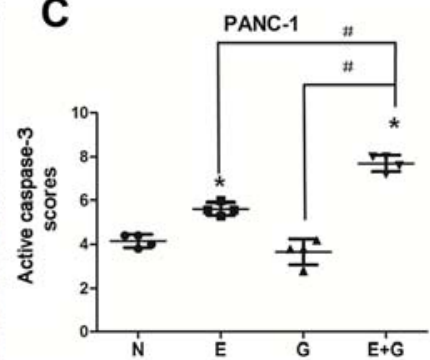

D

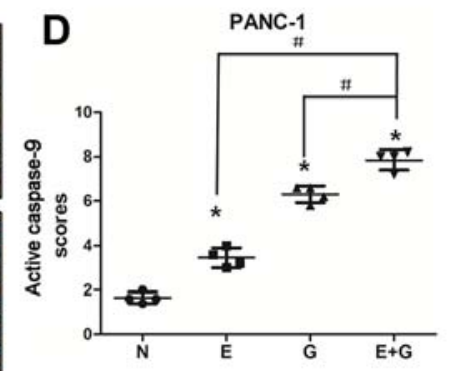

Figure 5. Gemcitabine in combination with erlotinib in recurrent tumors of PANC-1 cells induces increased activity of caspase-3 and caspase-9. (A and B) Immunohistochemical staining of active caspase- 9 and active caspase-3 in tumor tissue sections revealed active caspase- 9 activation by either the monotherapy and by the combination treatment and active-caspase-3 activation by erlotinib monotherapy and by the combination treatment in PANC-1 cells. Lower panels displayed higher magnification (original magnification, $\mathrm{x} 400$ ) of the boxed areas in the upper panels. Scale bars, $10 \mu \mathrm{m}$. (C and D) Visual fields (4-6) in each section of irradiation were photographed. The staining intensity and the percentage of positive cells were calculated and scored in each section. The total scores in each visual field were first analyzed using the homogeneity of variance test, followed by the change of variable test. The scores were presented as the mean $\pm \mathrm{SD}$. GraphPad Prism 5 was used to plot cartograms. ${ }^{*} \mathrm{P}<0.05$ indicates the controls vs. the treatment groups. ${ }^{\#} \mathrm{P}<0.05$ indicates the monotherapy (E or $\mathrm{G})$ vs. the combination $(\mathrm{E}+\mathrm{G})$ groups.

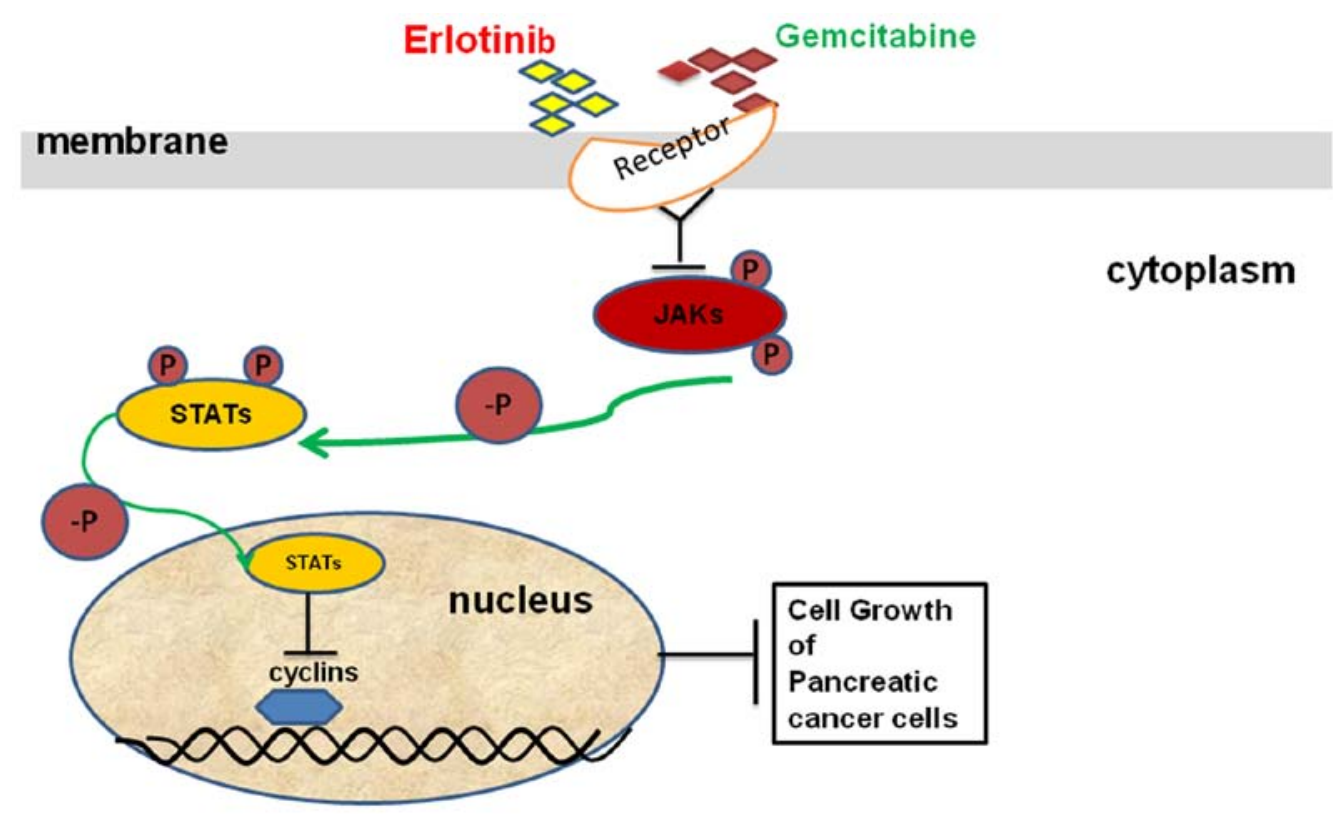

Figure 6. Schematic representation indicates that gemcitabine-erlotinib combination inhibits recurrent pancreatic tumor growth via JAK/STAT signaling. The gemcitabine-erlotinib combination significantly suppressed the phosphorylation levels of JAK1, JAK2, JAK3 as well as downstream STAT3 and STAT1 and eventually inhibited the growth of recurrent pancreatic tumors.

\section{Discussion}

In patients with pancreatic cancer following $\mathrm{R} 0$ or $\mathrm{R} 1$ resection, benefits from chemotherapy after surgery are usually very modest. Compared to single-agent gemcitabine treatment, gemcitabine in combination with erlotinib has been demonstrated to be effective for advanced pancreatic cancer. Nonetheless, its effectiveness in patients with PC after R0 or $\mathrm{R} 1$ resection has not yet been reported. Poor penetration of drugs into the dense and under vascularized tumor stroma in pancreatic cancer patients leads to poor response to chemotherapy. It has been reported that gemcitabine in 
combination with erlotinib is additive in the KRAS-mutated pancreatic cancer cells PANC-1, whereas it is antagonistic in the KRAS wild-type pancreatic cancer cell line BxPC-3 (16). However, the effect of gemcitabine in combination with erlotinib on recurrent tumor remains unknown. We hypothesized that gemcitabine in combination with erlotinib may also be effective for patients after resection due to better penetration of drugs into the tumor stroma. Herein, we explored its effect on recurrent tumors in a nude mouse model after resection. We found that gemcitabine in combination with erlotinib could inhibit tumor growth and induce apoptosis more effectively than gemcitabine or erlotinib alone, or the control group in vivo. The underlying mechanism may be JAK-STAT pathway inhibition induced by gemcitabine in combination with erlotinib.

As we aforementioned, the JAK-STAT pathway is very important in tumor proliferation. In the present study, we determined that the phosphorylation levels of JAK1, JAK2, JAK3 and their downstream STAT1 and STAT3 were significantly decreased in the E+G group. STAT1, a member of the family of STAT, has been reported to be expressed in $88 \%$ of primary pancreatic cancer patients (17). Reduced phospho-STAT1 has been reported to attenuate tumor growth and metastasis through downregulation of MUC4 mucin in pancreatic cancer (18). In our study, we revealed that gemcitabine in combination with erlotinib could lower the phosphorylation level of STAT1. These results revealed that gemcitabine in combination with erlotinib could also inhibit recurrent pancreatic cancer tumor growth by decreasing the phosphorylation levels of STAT1.

The phosphorylation of the tyrosine 705 residue in the STAT3 protein is a crucial event for its activation, that leads to formation of STAT3 homodimers and translocation into the nuclei (14). Nuclear localized STAT3 dimers bind to the promoters of various target genes, which are involved in cancer cell proliferation, survival and invasion, and regulate their transcription. (19). Nagaraj et al reported that gemcitabine in combination with erlotinib could not inhibit the phosphorylation of the serine 727 residue in STAT3 in vitro (20); while we found that gemcitabine in combination with erlotinib could inhibit the phosphorylation of tyrosine residue 705 in STAT3. Phosphorylation of tyrosine residue 705 or serine residue 727 in STAT3 may function differently, some genes may be preferentially regulated by only one or both phosphorylation of tyrosine residue 705 or serine residue 727 in STAT3 (21). The different roles that phosphorylation of tyrosine residue 705 or serine residue 727 in STAT3 may play in pancreatic cancer still remain unclear. We also determined that the expression of STAT3 downstream proteins, cyclin D1 and HIF-1 $\alpha$ were downregulated. Reduction in STAT3 phosphorylation at tyrosine residue 705 could cause activation of caspase- 9 and caspase-3, while apoptotic signaling could be mediated via activation of the cell death initiator procaspase-9, whereas activated caspase-9 in turn cleaves executioner caspase-3 $(15,20)$. The stroma of pancreatic cancer is often hypoxic, as indicated by the increased expression of HIF-1 $\alpha$ in stromal cells from human tumor sections, and HIF-1 $\alpha$ expression was revealed to be correlated with tumor size, worse prognosis, advanced stage, presence of lymph node metastasis, and a higher incidence of hepatic metastasis in pancreatic cancer (22). In addition, during a study that monitored HIF-1 $\alpha$ activity in vivo during tumor progression by using a human pancreatic cancer line engineered to express a reporter of HIF-1 $\alpha$ activity, the authors observed that the activity of HIF-1 $\alpha$ was accompanied by local invasion, peritoneal dissemination, and liver metastasis of pancreatic cells (23). Thus, a decreased expression level of HIF-1 $\alpha$ by gemcitabine in combination with erlotinib may also inhibit recurrent tumor growth.

The p53 protein is an important tumor-suppressor gene, which can arrest cell cycle progression at several points and induce apoptosis for cells undergoing uncontrolled growth (24). However, p53 mutants possess tumor-promoting functions (25). Loss or mutation of p53 occurs in $50 \%$ of human pancreatic cancers (26), including BxPC-3 and PANC-1 cells (27). It has been determined that STAT3 binds to the 53 promoter in vitro and in vivo, while blocking STAT3 in cancer cells upregulating the expression of p53, thus leading to p53-mediated tumor cell apoptosis (28). In the present study, we determined that reduced phosphorylation of STAT3 did not increase the expression of p53. Conversely, the expression of p53 was decreased in the gemcitabine in combination with erlotinib group. This may indicate that gemcitabine in combination with erlotinib may also inhibit tumor growth via downregulation of $\mathrm{p} 53$.

Cyclin D1 is an important regulator of cyclin-dependent kinase 4 (CDK4) and CDK6. It regulates cell cycle transition from the $\mathrm{G} 1$ to the $\mathrm{S}$ phase, where overexpression of cyclin D1 results in dysregulated CDK activity, rapid cell growth under restricted mitogenic signaling conditions, bypass of key cellular checkpoints, and ultimately, neoplastic growth (29). Hypoxia is most commonly present in the solid tumor microenvironment, and especially in pancreatic cancer. Hypoxia-inducible factor-1 (HIF-1; consists of highly regulated HIF-1a and a constitutively expressed HIF-1b) is the most important transcription factor resulting from intratumoral hypoxia, which can mediate many adaptive physiological responses $(22,30)$. In pancreatic cancer, HIF-1a expression levels are associated with tumor progression, fibrotic focus, angiogenesis, cell migration, and hepatic metastasis $(22,30)$. Cyclin D1 and HIF-1 $\alpha$ are both downstream proteins of STAT3. Decrease in the phosphorylation of STAT3 led to downregulation of cyclin D1 and HIF-1 $\alpha$, thus inhibiting tumor growth.

Our results indicated that gemcitabine in combination with erlotinib decreased the expression of p53 and the phosphorylation of tyrosine residue 705 in STAT3, which led to downregulation of cyclin D1 and HIF-1 $\alpha$ and activation of both caspase-9 and caspase-3. Downregulation of cyclin D1 and HIF-1 $\alpha$ contributed to the inhibition of G1/S cell cycle transition and angiogenesis. Activation of caspase- 9 and caspase-3 resulted in apoptosis of pancreatic cancer cells. Decreased expression of p53 could also decrease its tumorpromoting ability.

The heterogeneity of genetic alterations associated with pancreatic cancer emphasizes the need to identify whether the aforementioned treatment could function for pancreatic cancer cells of different genetic background. In the present study, we used two pancreatic cancer cell lines; BxPC-3 with wild-type KRAS, and PANC-1 with mutated type KRAS. KRAS mutation exists in almost $90 \%$ of pancreatic cancer cells, which leads to constitutive activation of Ras signaling and resistance to TKI $(31,32)$. Notably, we revealed that gemcitabine in combination with erlotinib could inhibit phosphorylation of 
tyrosine 705 residue in STAT3 in recurrent tumors of both cell lines. This suggests that inhibition of the phosphorylation of the 705 tyrosine residue in STAT3 by gemcitabine in combination with erlotinib does not depend on the state of KRAS.

Our results clearly revealed that the combination of gemcitabine and erlotinib could inhibit recurrent pancreatic tumor growth via downregulation of the phosphorylation levels of JAKs and STATs and as a result to induce apoptosis of pancreatic cancer cells (Fig. 6). Collectively, our findings provide compelling evidence for the use of gemcitabine in combination with erlotinib in the treatment of pancreatic cancer after $\mathrm{R} 0$ or $\mathrm{R} 1$ resection, and this combination may be used as a more effective treatment for pancreatic cancer after $\mathrm{R} 0$ or $\mathrm{R} 1$ resection than gemcitabine alone.

\section{Acknowledgements}

The present study was supported by Guizhou Province Science and Technology Projects [(2014)2015 and (2016)7232]. We would like to thank the Central Laboratory at Yongchuan Hospital, Chongqing Medical University and Clinical Research Center, Affiliated Hospital of Guizhou Medical University for housing all the experiments.

\section{References}

1. Siegel R, Naishadham D and Jemal A: Cancer statistics, 2013. CA Cancer J Clin 63: 11-30, 2013.

2. Hartwig W, Werner J, Jäger D, Debus J and Büchler MW: Improvement of surgical results for pancreatic cancer. Lancet Oncol 14: e476-e485, 2013.

3. Zhang Q, Zeng L, Chen Y, Lian G, Qian C, Chen S, Li J and Huang K: Pancreatic cancer epidemiology, detection, and management. Gastroenterol Res Pract 2016: 8962321, 2016.

4. Wang Y, Hu GF, Zhang QQ, Tang N, Guo J, Liu LY, Han X, Wang $\mathrm{X}$ and Wang $\mathrm{ZH}$ : Efficacy and safety of gemcitabine plus erlotinib for locally advanced or metastatic pancreatic cancer: A systematic review and meta-analysis. Drug Des Devel Ther 10: 1961-1972, 2016

5. Ducreux M, Cuhna AS, Caramella C, Hollebecque A, Burtin P, Goéré D, Seufferlein T, Haustermans K, Van Laethem JL, Conroy T, et al; ESMO Guidelines Committee: Cancer of the pancreas: ESMO Clinical Practice Guidelines for diagnosis, treatment and follow-up. Ann Oncol 26 (Suppl 5): v56-v68, 2015

6. Burris HA III, Moore MJ, Andersen J, Green MR, Rothenberg ML, Modiano MR, Cripps MC, Portenoy RK, Storniolo AM, Tarassoff $\mathrm{P}$, et al: Improvements in survival and clinical benefit with gemcitabine as first-line therapy for patients with advanced pancreas cancer: A randomized trial. J Clin Oncol 15: 2403-2413, 1997.

7. Binenbaum Y, Na'ara S and Gil Z: Gemcitabine resistance in pancreatic ductal adenocarcinoma. Drug Resist Updat 23: 55-68, 2015.

8. Bareschino MA, Schettino C, Troiani T, Martinelli E, Morgillo F and Ciardiello F: Erlotinib in cancer treatment. Ann Oncol 18 (Suppl 6): vi35-vi41, 2007.

9. Czarnecka AM, Korzeń P, Nowak-Dement A, Kukwa W, Korniluk J and Szczylik C: Prolonged complete response following gemcitabine-erlotinib combined therapy in advanced pancreatic cancer. Oncol Lett 11: 1101-1104, 2016.

10. Rawlings JS, Rosler KM and Harrison DA: The JAK/STAT signaling pathway. J Cell Sci 117: 1281-1283, 2004.

11. Mace TA, Shakya R, Elnaggar O, Wilson K, Komar HM, Yang J, Pitarresi JR, Young GS, Ostrowski MC, Ludwig T, et al: Single agent BMS-911543 Jak2 inhibitor has distinct inhibitory effects on STAT5 signaling in genetically engineered mice with pancreatic cancer. Oncotarget 6: 44509-44522, 2015.

12. Egberts JH, Cloosters V, Noack A, Schniewind B, Thon L, Klose S, Kettler B, von Forstner C, Kneitz C, Tepel J, et al: Anti-tumor necrosis factor therapy inhibits pancreatic tumor growth and metastasis. Cancer Res 68: 1443-1450, 2008.
13. Naito S, von Eschenbach AC, Giavazzi R and Fidler IJ: Growth and metastasis of tumor cells isolated from a human renal cell carcinoma implanted into different organs of nude mice. Cancer Res 46: 4109-4115, 1986.

14. Huang G, Yan H, Ye S, Tong C and Ying QL: STAT3 phosphorylation at tyrosine 705 and serine 727 differentially regulates mouse ESC fates. Stem Cells 32: 1149-1160, 2014.

15. Agarwal C, Tyagi A, Kaur M and Agarwal R: Silibinin inhibits constitutive activation of Stat3, and causes caspase activation and apoptotic death of human prostate carcinoma DU145 cells. Carcinogenesis 28: 1463-1470, 2007.

16. Torres C, Linares A, Alejandre MJ, Palomino-Morales RJ, Delgado JR and Perales S: Interplay between gemcitabine and erlotinib over pancreatic adenocarcinoma cells. Pancreas 45: 269-280, 2016.

17. Sun Y, Yang S, Sun N and Chen J: Differential expression of STAT1 and p21 proteins predicts pancreatic cancer progression and prognosis. Pancreas 43: 619-623, 2014.

18. Seshacharyulu P, Ponnusamy MP, Rachagani S, Lakshmanan I, Haridas D, Yan Y, Ganti AK and Batra SK: Targeting EGF-receptor(s) - STAT1 axis attenuates tumor growth and metastasis through downregulation of MUC4 mucin in human pancreatic cancer. Oncotarget 6: 5164-5181, 2015.

19. Ye C, Zhao W, Li M, Zhuang J, Yan X, Lu Q, Chang C, Huang X, Zhou J, Xie B, et al: $\delta$-tocotrienol induces human bladder cancer cell growth arrest, apoptosis and chemosensitization through inhibition of STAT3 pathway. PLoS One 10: e0122712, 2015.

20. Nagaraj NS, Washington MK and Merchant NB: Combined blockade of Src kinase and epidermal growth factor receptor with gemcitabine overcomes STAT3-mediated resistance of inhibition of pancreatic tumor growth. Clin Cancer Res 17: 483-493, 2011.

21. Ouédraogo ZG, Biau J, Kemeny JL, Morel L, Verrelle P and Chautard E: Role of STAT3 in genesis and progression of human malignant gliomas. Mol Neurobiol 54: 5780-5797, 2017.

22. Yuen A and Díaz B: The impact of hypoxia in pancreatic cancer invasion and metastasis. Hypoxia (Auckl) 2: 91-106, 2014.

23. Kizaka-Kondoh S, Itasaka S, Zeng L, Tanaka S, Zhao T, Takahashi Y, Shibuya K, Hirota K, Semenza GL and Hiraoka M: Selective killing of hypoxia-inducible factor-1-active cells improves survival in a mouse model of invasive and metastatic pancreatic cancer. Clin Cancer Res 15: 3433-3441, 2009.

24. Sun C, Zhang M, Shan X, Zhou X, Yang J, Wang Y, Li-Ling J and Deng Y: Inhibitory effect of cucurbitacin E on pancreatic cancer cells growth via STAT3 signaling. J Cancer Res Clin Oncol 136: 603-610, 2010.

25. Stojanovic N, Hassan Z, Wirth M, Wenzel P, Beyer M, Schäfer C Brand P, Kroemer A, Stauber RH, Schmid RM, et al: HDAC1 and HDAC2 integrate the expression of p53 mutants in pancreatic cancer. Oncogene 36: 1804-1815, 2017.

26. Rosenfeldt MT, O'Prey J, Morton JP, Nixon C, MacKay G, Mrowinska A, Au A, Rai TS, Zheng L, Ridgway R, et al: p53 status determines the role of autophagy in pancreatic tumour development. Nature 504: 296-300, 2013.

27. Izetti P, Hautefeuille A, Abujamra AL, de Farias CB, Giacomazzi J, Alemar B, Lenz G, Roesler R, Schwartsmann G, Osvaldt AB, et al: PRIMA-1, a mutant $\mathrm{p} 53$ reactivator, induces apoptosis and enhances chemotherapeutic cytotoxicity in pancreatic cancer cell lines. Invest New Drugs 32: 783-794, 2014.

28. Niu G, Wright KL, Ma Y, Wright GM, Huang M, Irby R, Briggs J, Karras J, Cress WD, Pardoll D, et al: Role of Stat 3 in regulating p53 expression and function. Mol Cell Biol 25: 7432-7440, 2005.

29. Qie S and Diehl JA: Cyclin D1, cancer progression, and opportunities in cancer treatment. J Mol Med (Berl) 94: 1313-1326, 2016.

30. Zhao X, Gao S, Ren H, Sun W, Zhang H, Sun J, Yang S and Hao J: Hypoxia-inducible factor-1 promotes pancreatic ductal adenocarcinoma invasion and metastasis by activating transcription of the actin-bundling protein fascin. Cancer Res 74: 2455-2464, 2014.

31. Berry W, Algar E, Kumar B, Desmond C, Swan M, Jenkins BJ and Croagh D: Endoscopic ultrasound-guided fine-needle aspirate-derived preclinical pancreatic cancer models reveal panitumumab sensitivity in KRAS wild-type tumors. Int J Cancer 140: 2331-2343, 2017.

32. Zehir A, Benayed R, Shah RH, Syed A, Middha S, Kim HR, Srinivasan P, Gao J, Chakravarty D, Devlin SM, et al: Mutational landscape of metastatic cancer revealed from prospective clinical sequencing of 10,000 patients. Nat Med 23: 703-713, 2017.

This work is licensed under a Creative Commons Attribution-NonCommercial-NoDerivatives 4.0 International (CC BY-NC-ND 4.0) License. 